\title{
Disturbance Attenuation for a Class of Exponentially Uncertain Switched Linear Systems with State Delay
}

\author{
Hongmei Huang ${ }^{1,2}$, Fei Long ${ }^{2}$, Changlin $\mathrm{Li}^{2}$, Adang Ding ${ }^{2,3}$ \\ ${ }^{1}$ College of Science, Guizhou Institute of Technology, Guiyang, Guizhou. 550003. China \\ ${ }^{2}$ Institute of Intelligent Information Processing, Guizhou University, Guiyang, Guizhou \\ 550025. China \\ ${ }^{3}$ Faculty of Information Engineering, Guizhou Institute of Technology, Guiyang, Guizhou \\ clin817@163.com
}

\begin{abstract}
In this paper, we address the disturbance attenuation problem for a class of state delay switched linear systems with exponential uncertainties via switched state feedback and switched dynamic output feedback, respectively. By using Taylor series approximation and convex polytope technique, exponentially uncertain switched linear systems with state delay is transformed into an equivalent switched polytopic model with additive norm bounded uncertainty. For such equivalent switched model, we design its switching strategy and associated state feedback subcontrollers or dynamic output feedback sub-controllers so that whole switched model is asymptotical stabilization with $H_{\infty}$ disturbance attenuation based on multiple Lyapunov function technology and LMI approach.
\end{abstract}

Keywords: Switched Linear Systems with State Delay; Exponential Uncertainty; Disturbance Attenuation; State Feedback; Dynamic Output Feedback; LMIs

\section{Introduction}

As we know, state delay is always in practical control system due to the action speed limitation of mechanism and electronics. Such as paper-making process, chemical process, long transmission lines in pneumatic systems and communication channels, etc. On the one hand, the phenomenon of state delay is commonly the source of instability and bad-performance in practical; on the other hand, due to the state delay, it is getting more difficult and complex to control such systems effectively. Hence, analysis and synthesis system with state delay is always the hot spot issues of research field in the control theory and control engineering. In recent decade, the linear systems with state delay have been extensively investigated [1-3]. However, so far there have been few results for switched linear systems with state delay. [4, 5] studied the controllability of switched linear systems with state delay and discrete-time switched linear systems with state delay, respectively. [6] considered the stabilization of switched linear systems with state delay based on convex combination technique. [7] addressed the method of delay-dependent robust H-infinity control for a class of uncertain switched systems with state delay, however, in this case, the condition of matrix inequality is not linear matrix inequality, its solving have to make use of iterative method or enforced constraint. Besides, [8-10] studied the stability and stabilization for a class of switched linear systems with state delay.

In order to design a computer based control for switched linear system (whose coefficient matrix is $M_{\sigma}$ and $N_{\sigma}$ ), L. Hetel, et al., [11, 12] show that sampled model of system is derived and discrete time control methods are applied. Under the case that sampling and actuation are periodic and synchronous with the periodicity, the coefficient matrix of the sampled model is given by 


$$
A_{\sigma}=e^{M_{\sigma} \rho}, B_{\sigma}=\left(\int_{0}^{\rho} e^{M_{\sigma} \tau} d \tau\right) N_{\sigma}
$$

where $\rho$ is sampling periodicity. It is well know that, in many control problems, the sampling periodicity of system is often affected by some delays (delays between the sensor and the digital control, computing delays in the controller, communication delays between the controller and the actuator, and so on). Furthermore, these delays are often unknown, time-varying and bounded [13]. Therefore, exponential uncertainty is inevitable in the process of modeling system. Hence, the control synthesis of switched linear systems with state delay subject to exponential uncertainties is a very important and challenging problem because of the practical background of exponential uncertainty.

Generally speaking, exponential uncertainty represents the terms like $e^{M \rho}$ or $\int_{0}^{\rho} e^{M \tau} d \tau$ that depend on an unknown, possibly time-varying and bounded parameter $\rho$. In the literature [14], exponential uncertainty is treated by assuming estimable delay uncertainties. Andrea Balluchi, et al., [15] treats the uncertain exponential terms as bounded uncertainties. Hetel, et al., [11, 12] introduces exponential uncertainties to hybrid linear system and treats the uncertain exponential terms as polytopic uncertainties. Under the arbitrary switching rule, Hetel, et al., [11] design state feedback sub-controller for the stabilization problem of a class of hybrid linear systems with exponential uncertainties in the case where the switching and the sampling are synchronous. And then the obtained results in the literature [11] are extended to cope with network controlled systems [12]. In this manuscript, Motivated by the reference [11, 12], we intend to investigate the disturbance attenuation problem for a class of exponentially uncertain switched linear systems with state delay. Throughout this note, it is assumed that the switching strategy is picked in such a way that there are finite switches in finite time and the state of system does not jump in the instantaneous switching.

Our goal is, for a class of state delay switched linear systems subject to exponential uncertainty, to design a switching strategy $\sigma(t)$ and associated state feedback sub-controllers or dynamic output feedback sub-controllers such that the resulting closed-loop system is asymptotical stabilization with a prescribed $H_{\infty}$ disturbance attenuation level for all admissible uncertainties. Firstly, we show that exponentially uncertain switched system with state delay is transformed into an equivalent polytopic model with an additive norm bounded uncertainty based on Taylor series approximation and convex polytope technique. And then, by taking advantage of multiple Lyapunov function technology and LMI approach, the robust $H_{\infty}$ disturbance attenuation property of such equivalent switched model is investigated via switched state feedback and switched dynamic output feedback, respectively.

The remainder of this paper is organized as follows: The problem statement and some preliminaries are described in Section 2, while in Section 3 the asymptotical stabilization with $H_{\infty}$ disturbance attenuation for exponentially uncertain switched linear system with state delay is investigated via switched state feedback. In Section 4, we discuss the asymptotical stabilization with $H_{\infty}$ disturbance attenuation for a class of state delay switched linear systems subject to exponential uncertainties via switched dynamic output feedback. Two numerical examples are presented in Section 5 to illustrate our results. Finally, some conclusions are drawn in Section 6.

Notations: We use standard notations throughout this paper. Matrix $P^{T}$ stands for the transpose of the matrix $P \cdot L_{2}[0, T](0 \leq T<\infty)$ denotes the space of square integrable functions on $[0, T]$ and

$$
\|\omega\|_{L_{2}[0, T]}=\left(\int_{0}^{T} \omega^{T}(t) \omega(t) d t\right)^{1 / 2}, \forall \omega \in L_{2}[0, T]
$$


The symmetric terms in a symmetric matrix are denoted by*, $\mathfrak{R}^{\perp}$ denote any matrix whose columns form bases of the null space of $\Re$ and $\lambda_{\min }(P)$ denote the minimum eigenvalues of a symmetric matrix $P$.

\section{Problem Statements and Some Lemmas}

In this paper, based on multiple Lyapunov function technology and LMI approach, we investigate the asymptotical stabilization with $H_{\infty}$ disturbance attenuation for the exponentially uncertain state delay switched linear systems (1) and (2) via switched state feedback and switched dynamic output feedback, respectively.

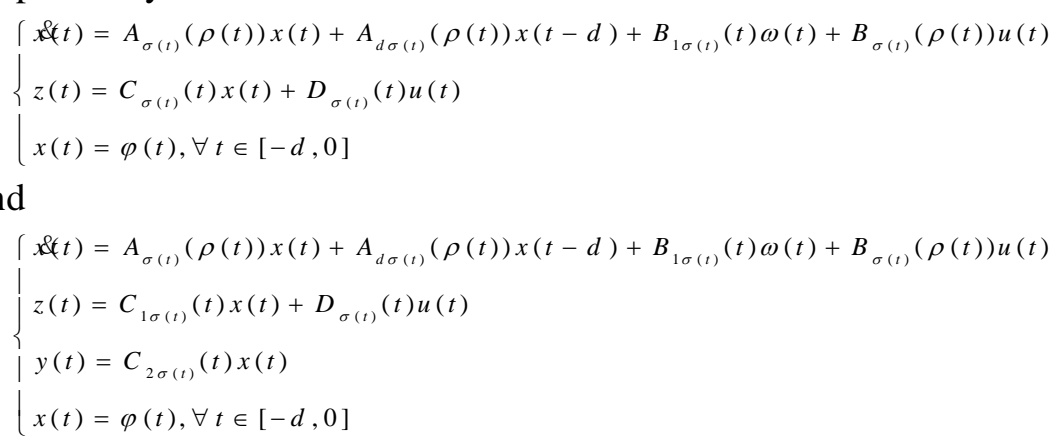

where $x \in \mathrm{i}^{n}$ is the system state; $u \in \mathrm{i}^{m}$ is the continuous control input; $\omega \in \mathrm{i}^{\prime}$ is the exogenous disturbance input that belongs to $L_{2}[0,+\infty] ; y \in i^{p}$ is the measured output; $z \in \mathrm{i}^{q}$ is the regulated output; $d>0$ is a constant positive delay, $\varphi(t) \in \square\left([-d, 0],{ }^{n}\right)$ is a given continuous vector valued initial function. The switching signal $\sigma(\cdot):[0, \infty) \rightarrow$ 쩖 $=\{1,2,, N\}, N<\infty$ stands for the piecewise constant switching strategy to be designed. $A_{\sigma}(\rho(t))=e^{M_{\sigma} \rho(t)}, A_{d \sigma}(\rho(t))=\left(\int_{0}^{\rho(t)} e^{M_{\sigma} s} d s\right) R_{\sigma}, B_{\sigma}(\rho(t))=\left(\int_{0}^{\rho(t)} e^{M_{\sigma} s} d s\right) N_{\sigma}$. Gives a particular index $i$ indicating the active system regime. The uncertain parameter $\rho(t)$ is positive, time varying, bounded and $0<\underline{\rho}<\rho(t)<\bar{\rho}$. $M_{i} \in \mathrm{i}^{n \times n}, R_{i} \in \mathrm{i}^{n \times n}$ and $N_{i} \in \mathrm{i}^{n \times m}$ are three families of matrices, $B_{1 i}, C_{i}, C_{1 i}, C_{2 i}$ and $D_{i}$ are constant matrices with appropriate dimensions $(i \in \bar{¥})$.

The objective of this paper is, for any given $\gamma>0$, to find $\mathrm{N}$ switched state feedback controllers $u(t)=K_{i} x(t), i=1,2, \cdots N$ ( or $N$ switched output feedback controllers $\left.u(t)=K_{i}(s) y, i=1,2, \cdots N\right)$ so that the state delay switched linear system (1) ( or the state delay switched linear system (2) ) satisfies:

a) With zero disturbance input condition $\omega \equiv 0$, it is asymptotically stable for all admissible uncertainties.

b) With initial condition $x(t)=0, \forall t \in[-d, 0], \int_{0}^{T} z^{T}(t) z(t) d t<\gamma^{2} \int_{0}^{T} \omega^{T}(t) \omega(t) d t$ for all nonzero $\omega \in L_{2}[0, T](0 \leq T<\infty)$ and all admissible uncertainties.

Now, we introduce some support lemmas and the concept of switching sequence, which can be use in the later.

Lemma 2.1 ([12]): Consider the uncertain polynomial parameter dependent $n$ order matrix

$$
L(\rho)=L_{0}+L_{1} \rho+L_{2} \rho^{2}+\mathrm{L}+L_{h} \rho^{h}
$$

with the uncertain parameter $\rho$ is positive, bounded and $0<\underline{\rho}<\rho<\bar{\rho}$ (where $\underline{\rho}$ and $\bar{\rho}$ are known constants). Then we can find a convex polytope with $h+1$ vertices that 
envelopes

$$
L(\rho)
$$

i.e.

there

exist

parameters $\mu_{j}(\rho), j=1,2, \mathrm{~L}, h+1$ satisfying $\sum_{j=1}^{h+1} \mu_{j}(\rho)=1, \mu_{j}(\rho)>0$ such that

$$
L(\rho)=\sum_{j=1}^{h+1} \mu_{j}(\rho) U U_{j}
$$

where $U_{j}, j=1,2, \mathrm{~L}, h+1$ represent the polytope vertices given as follows.

$$
\left\{\begin{array}{l}
U_{1}=L_{0}+L_{1} \underline{\rho}+L_{2} \underline{\rho}^{2}+\mathrm{L}+L_{h} \underline{\rho}^{h}, U_{2}=L_{0}+L_{1} \bar{\rho}+L_{2} \underline{\rho}^{2}+\mathrm{L}+L_{h} \underline{\rho}^{h} \\
U_{3}=L_{0}+L_{1} \bar{\rho}+L_{2} \bar{\rho}^{2}+\mathrm{L}+L_{h} \underline{\rho}^{h}, \mathrm{~L}, U_{h+1}=L_{0}+L_{1} \bar{\rho}+L_{2} \bar{\rho}^{2}+\mathrm{L}+L_{h} \bar{\rho}^{h}
\end{array}\right.
$$

The relation between the uncertain parameter $\rho$ and $\mu_{j}(\rho)$ is given by

$$
\mu_{j}(\rho)= \begin{cases}1-(\rho-\underline{\rho}) /(\bar{\rho}-\underline{\rho}), & j=1 \\ \left(\rho^{j-1}-\underline{\rho}^{j-1}\right) /\left(\bar{\rho}^{j-1}-\underline{\rho}^{j-1}\right)-\left(\rho^{j}-\underline{\rho}^{j}\right) /\left(\bar{\rho}^{j}-\underline{\rho}^{j}\right), j=2,3, \mathrm{~L}, h \\ \left(\rho^{h}-\underline{\rho}^{h}\right) /\left(\bar{\rho}^{h}-\underline{\rho}^{h}\right), & j=h+1\end{cases}
$$

Lemma 2.2 (Schur complement [16]): For constant matrices $M, L$ and $Q$ with appropriate dimensions where $M$ and $Q$ are symmetric and $Q>0$, then $M+L^{T} Q L<0$ if and only if

$$
\left(\begin{array}{cc}
M & L^{T} \\
L & -Q^{-1}
\end{array}\right)<0 \text { or } \quad\left(\begin{array}{cc}
-Q^{-1} & L \\
L^{T} & M
\end{array}\right)<0
$$

Lemma 2.3 ([17]): Given a symmetric matrix $\Psi \in \square^{n \times n}$ and two matrices $\Gamma, \Xi$ of column dimension $\mathrm{m}$, consider the problem of finding some matrix $\Theta$ of compatible dimensions such that

$$
\Psi+\Gamma^{T} \Theta^{T} \Xi+\Xi^{T} \Theta \Gamma<0
$$

Then the above matrix inequality is solvable for $\Theta$ if and only if

$$
\left\{\begin{array}{l}
\Gamma^{\perp T} \Psi \Gamma^{\perp}<0 \\
\Xi^{\perp T} \Psi \Xi^{\perp}<0
\end{array}\right.
$$

Lemma 2.4([17]): Given symmetrically positive definite matrices $x \in \square^{n \times n}$ and $Y \in \square^{n \times n}, n_{k}$ is a positive integer, then there exist matrices $X_{2}, Y_{2} \in \square^{n \times n_{k}}$ and symmetrically matrices $X_{3}, Y_{3} \in \square^{n_{k} \times n_{k}}$ are satisfied

if and only if

$$
\left[\begin{array}{cc}
X & X_{2} \\
X_{2}^{T} & X_{3}
\end{array}\right]>0,\left[\begin{array}{cc}
X & X_{2} \\
X_{2}^{T} & X_{3}
\end{array}\right]^{-1}=\left[\begin{array}{cc}
Y & Y_{2} \\
Y_{2}^{T} & Y_{3}
\end{array}\right]
$$

$$
\left[\begin{array}{cc}
X & I \\
I & Y
\end{array}\right] \geq 0, \operatorname{rank}\left[\begin{array}{cc}
X & I \\
I & Y
\end{array}\right] \leq n+n_{k}
$$

Definition2.1 (switching sequence): The sequence $\left\{\left(t_{m}, r_{m}\right) \mid r_{m} \in \bar{\square} ; m=1,2, \cdots\right\}$ is said to be switching sequence, if

$$
\begin{aligned}
& \text { (i): } \sigma\left(t_{m}^{-}\right) \neq \sigma\left(t_{m}^{+}\right), \\
& \text {(ii): } \sigma(t)=\sigma\left(t_{m}^{+}\right)=r_{m}, t \in\left[t_{m}, t_{m+1}\right)
\end{aligned}
$$

Moreover, the constant $t_{m}$ is said to be dwell time length of the $r_{m}-t h$ subsystem.

\section{Robust $H_{n}$ Control via Switched State Feedback}

In this section, the robust $H_{\infty}$ problem of state delay switched linear system (1) is investigated via switched state feedback. Before the design of switched stabilization controller, we firstly show how the system (1) can be expressed as a switched polytopic system with an additive norm bounded uncertainty. Such a formulation makes the robust stabilization problem tractable.

According to the Lemma 2.1 and the properties of exponential matrix, 
The following Lemma is obvious.

$$
e^{M x}=\sum_{i=0}^{\infty} \frac{M^{i}}{i !} x^{i}, \quad \int_{0}^{x} e^{M s} d s=\sum_{i=1}^{\infty} \frac{M^{i-1}}{i !} x^{i}
$$

Lemma 3.1: The state delay switched linear system (1) subject to exponential uncertainties can be expressed as:

$$
\left\{\begin{aligned}
x \in(t)= & \left(A_{\sigma}^{h}(\rho(t))+\Delta A_{\sigma}^{h}(\rho(t))\right) x(t)+\left(A_{d \sigma}^{h}(\rho(t))+\Delta A_{d \sigma}^{h}(\rho(t))\right) x(t-d) \\
& +\left(B_{\sigma}^{h}(\rho(t))+\Delta B_{\sigma}^{h}(\rho(t))\right) u(t)+B_{1 \sigma} \omega(t) \\
z(t)= & C_{\sigma} x(t)+D_{\sigma} u(t) \\
x(t)= & \varphi(t), \forall t \in[-d, 0]
\end{aligned}\right.
$$

where

$$
\begin{aligned}
& A_{\sigma}^{h}(\rho(t))=\sum_{j=0}^{h} \frac{M_{\sigma}^{j}}{j !} \rho^{j}(t)=\sum_{j=1}^{h+1} \mu_{j}(\rho(t)) U_{\sigma j}^{A h} \\
& A_{d \sigma}^{h}(\rho(t))=\left(\sum_{j=1}^{h} \frac{M_{\sigma}^{j-1}}{j !} \rho^{j}(t)\right) R_{\sigma}=\left(\sum_{j=1}^{h+1}\left(\mu_{j}(\rho(t)) U_{\sigma j}^{B h}\right)\right) R_{\sigma} \\
& B_{\sigma}^{h}(\rho(t))=\left(\sum_{j=1}^{h} \frac{M_{\sigma}^{j-1}}{j !} \rho^{j}(t)\right) N_{\sigma}=\left(\sum_{j=1}^{h+1}\left(\mu_{j}(\rho(t)) U_{\sigma j}^{B h}\right)\right) N_{\sigma} \\
& \sum_{j=1}^{h+1} \mu_{j}(\rho)=1, \mu_{j}(\rho)>0, j=1,2, \mathrm{~L}, h+1 .
\end{aligned}
$$

$$
\left\{\begin{array}{l}
U_{\sigma 1}^{B h}=\bar{\rho} I+\frac{M_{\sigma}}{2 !} \underline{\rho}^{2}+\mathrm{L}+\frac{M_{\sigma}^{h}}{(h+1) !} \underline{\rho}^{h+1}, \quad U_{\sigma 2}^{B h}=\bar{\rho} I+\frac{M_{\sigma}}{2 !} \bar{\rho}^{2}+\mathrm{L}+\frac{M_{\sigma}^{h}}{(h+1) !} \underline{\rho}^{h+1}, \\
U_{\sigma 3}^{B h}=\bar{\rho} I+\frac{M_{\sigma}}{2 !} \bar{\rho}^{2}+\mathrm{L}+\frac{M_{\sigma}^{h}}{(h+1) !} \underline{\rho}^{h+1}, \mathrm{~L}, U_{\sigma, h+1}^{B h}=\bar{\rho} I+\frac{M_{\sigma}}{2 !} \bar{\rho}^{2}+\mathrm{L}+\frac{M_{\sigma}^{h}}{(h+1) !} \bar{\rho}^{h+1}
\end{array}\right.
$$

The remainders of the Taylor series approximation $\Delta A_{\sigma}^{h}(\rho(t)), \Delta A_{d \sigma}^{h}(\rho(t))$ and $\Delta B_{\sigma}^{h}(\rho(t))$, are given as follows:

$$
\left\{\begin{array}{l}
\Delta A_{\sigma}^{h}(\rho(t))=e^{M_{\sigma} \rho(t)}-\sum_{j=0}^{h} \frac{M_{\sigma}^{j}}{j !} \rho^{j}(t) \\
\Delta A_{d \sigma}^{h}(\rho(t))=\left(\int_{0}^{\rho(t)} e^{M_{\sigma} s} d s-\sum_{j=1}^{h+1} \frac{M_{\sigma}^{j-1}}{j !} \rho^{j}(t)\right) R_{\sigma} \\
\Delta B_{\sigma}^{h}(\rho(t))=\left(\int_{0}^{\rho(t)} e^{M_{\sigma} s} d s-\sum_{j=1}^{h+1} \frac{M_{\sigma}^{j-1}}{j !} \rho^{j}(t)\right) N_{\sigma}
\end{array}\right.
$$

The relation between the uncertain parameter $\rho(t)$ and the coordinates $\mu_{j}(\rho)$ is given by lemma 2.1 .

Notice that the uncertain items $\Delta A_{\sigma}^{h}(\rho(t)), \Delta A_{d \sigma}^{h}(\rho(t))$ and $\Delta B_{\sigma}^{h}(\rho(t))$ are bounded while $0<\underline{\rho}<\rho(t)<\bar{\rho}$. Therefore one can write

$$
\left\|\Delta A_{\sigma}^{h}(\rho(t))\right\| \leq \gamma_{A},\left\|\Delta A_{d \sigma}^{h}(\rho(t))\right\| \leq \gamma_{A_{d}},\left\|\Delta B_{\sigma}^{h}(\rho(t))\right\| \leq \gamma_{B}
$$

where 


$$
\left\{\begin{array}{l}
\gamma_{A}=\sup _{\underline{\rho}<\rho(t)<\bar{\rho}} \max ^{-1 \leq i \leq N}\left\|e^{M_{i} \rho(t)}-\sum_{j=0}^{h} \frac{M_{i}^{j}}{j !} \rho^{j}(t)\right\| \\
\gamma_{A_{d}}=\sup _{\underline{\rho}<\rho(t)<\bar{\rho}} \max _{1 \leq i \leq N}\left\|\left(\int_{0}^{\rho(t)} e^{M_{i} s} d s-\sum_{j=1}^{h+1} \frac{M_{i}^{j-1}}{j !} \rho^{j}(t)\right) R_{i}\right\| \\
\gamma_{B}=\sup _{\underline{\rho}<\rho(t)<\bar{\rho}-1 \leq i \leq N} \max _{0}\left\|\left(\int_{0}^{\rho(t)} e^{M_{i} s} d s-\sum_{j=1}^{h+1} \frac{M_{i}^{j-1}}{j !} \rho^{j}(t)\right) N_{i}\right\|
\end{array}\right.
$$

Remark 3.1: the describing and proof of lemma 3.1 is come from the same idea of literature [12].

By the above analysis, switched dynamic model (3) is actually a switched polytopic system subject to an additive norm bounded uncertainty. As lemma 3.1 has showed, such a switched model can be used to represent the state delay switched system (1). Therefore, stabilizing switched dynamic model (3) is equivalent to doing the system (1).

Our goal in this section, for the uncertain state delay switched linear system (3), is to investigate stabilization with $H_{\infty}$ disturbance attenuation level $\gamma$ via switched state feedback. It is stated as follows: for any given $\gamma>0$, design a switching strategy $\sigma(t)$ and $N$ associated state feedback sub-controller $u(t)=K_{i}(t) x(t),(i=1,2, \cdots N)$ such that the resulting closed-loop system (3) satisfies the following:

a) With zero disturbance input condition $\omega \equiv 0$, it is asymptotically stable for all admissible uncertainties.

b) With initial condition $x(t)=0, \forall t \in[-d, 0], \int_{0}^{T} z^{T}(t) z(t) d t<\gamma^{2} \int_{0}^{T} \omega^{T}(t) \omega(t) d t$ for all nonzero $\omega \in L_{2}[0, T](0 \leq T<\infty)$ and all admissible uncertainties.

For disturbance attenuation performance of system (3), we have the following result.

Theorem 3.1: Given any constant $\gamma>0$, the uncertain state delay switched linear system (3) is asymptotically stabilization with $H_{\infty}$ disturbance attenuation $\gamma$ via switched state feedback if there exist symmetrically positive definite matrices $x_{i}$ and matrices $Y_{i}$ with $i \in \bar{\nexists}$ such that the following linear matrix inequality is satisfied for any $i=1,2, \mathrm{~L}, N$.

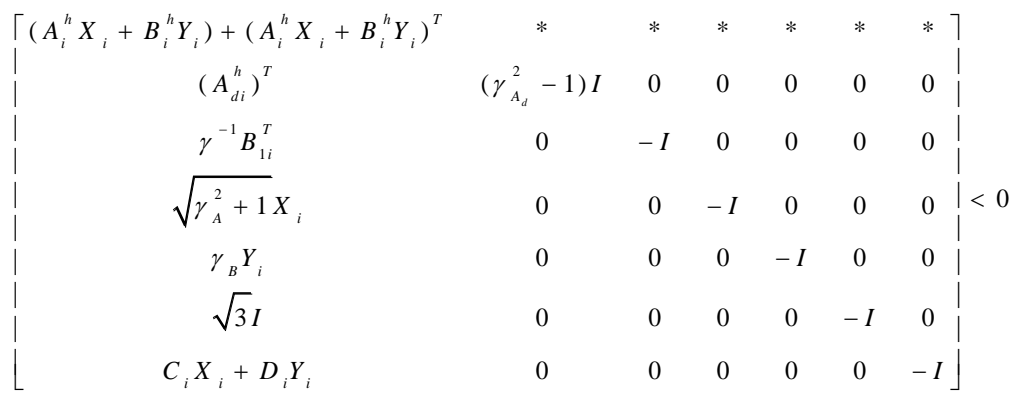

In this case, the state feedback sub-controller gain and switching strategy are taken as

$$
\left\{\begin{array}{l}
K_{i}=Y_{i} X_{i}^{-1} \\
\sigma(k)=\arg \min _{i \in \bar{F}} x^{T}(t) \mid \begin{array}{l}
X_{i}^{-1} A_{C i}+A_{C i}^{T} X_{i}^{-1}+\left(1-\gamma_{A_{d}}^{2}\right)^{-1} X_{i}^{-1} A_{d i}^{h}\left(A_{d i}^{h}\right)^{T} X_{i}^{-1} \\
\left\lfloor+\gamma^{-2} X_{i}^{-1} B_{1 i} B_{1 i}^{T} X_{i}^{-1}+\left(\gamma_{A}^{2}+1\right) I+\gamma_{B}^{2} K_{i}^{T} K_{i}+3 X_{i}^{-2}+C_{C i}^{T} C_{C i}\right\rfloor
\end{array}
\end{array}\right.
$$

where

$$
A_{C i}(\rho(t))=A_{i}^{h}(\rho(t))+B_{i}^{h}(\rho(t)) K_{i}, C_{C i}=C_{i}+D_{i} K_{i} .
$$

Proof: Setting $X_{i}=P_{i}^{-1}, Y_{i}=K_{i} P_{i}^{-1}$, then the matrix inequality (13) is equivalent with the following matrix inequality 


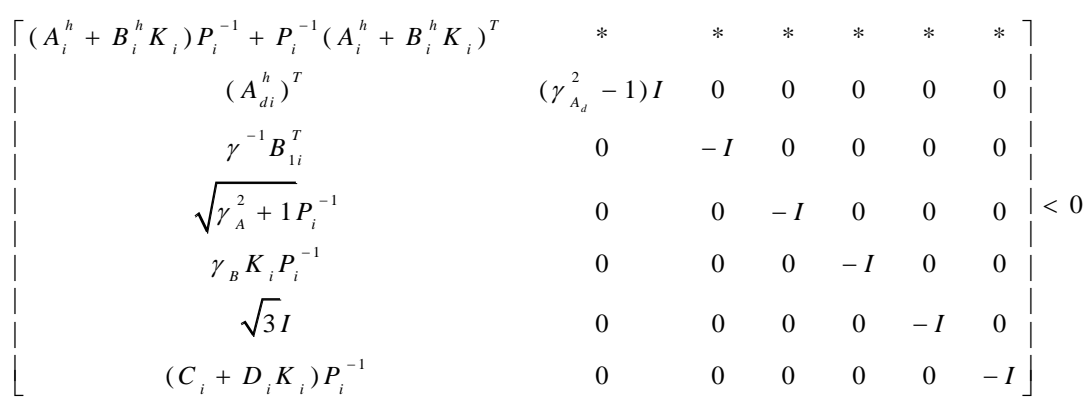

Pro-multiplying and post-multiplying the matrix diag $\left(P_{i}, I, I, I, I, I, I\right)$ in left-side of matrix inequality (15), the matrix inequality implies the following matrix inequality.

$$
\left\{\begin{array}{ccccccc}
P_{i} A_{C i}+A_{C i}^{T} P_{i} & * & * & * & * & * & * \\
\left(A_{d i}^{h}\right)^{T} P_{i} & \left(\gamma_{A_{d}}^{2}-1\right) I & 0 & 0 & 0 & 0 & 0 \\
\gamma^{-1} B_{1 i}^{T} P_{i} & 0 & -I & 0 & 0 & 0 & 0 \\
\sqrt{\gamma_{A}^{2}+1 I} & 0 & 0 & -I & 0 & 0 & 0 \\
\gamma_{B} K_{i} & 0 & 0 & 0 & -I & 0 & 0 \\
\sqrt{3} P_{i} & 0 & 0 & 0 & 0 & -I & 0 \\
C_{C i} & 0 & 0 & 0 & 0 & 0 & -I
\end{array}\right]<0
$$

In view of Lemma 2.2, the matrix inequality (16) is equivalent to the following inequality.

$$
\left[\begin{array}{cc}
P_{i} A_{C i}+A_{C i}^{T} P+\gamma^{-2} P_{i} B_{1 i} B_{1 i}^{T} P_{i}+\left(\gamma_{A}^{2}+1\right) I+\gamma_{B}^{2} K_{i}^{T} K_{i}+3 P_{i}^{2}+C_{C i}^{T} C_{C i} & P_{i} A_{d i}^{h} \\
\left(A_{d i}^{h}\right)^{T} P_{i} & \left(\gamma_{A_{d}}^{2}-1\right) I
\end{array}\right]<0
$$

Consider the notations:

$$
\begin{gathered}
\alpha_{i}(t)= \begin{cases}1 & t \in \Omega_{i} \\
0 & t \notin \Omega_{i}\end{cases} \\
\Omega_{i}=\{t \mid \text { the i-th subsystem is active at time instant } t\}, i=1,2, \cdots, N .
\end{gathered}
$$

The closed-loop dynamic of system (3) with state feedback controller $u(t)=K_{\sigma(t)} x(t)$ is given by:

$$
\left\{\begin{array}{l}
x(t)=\left(A_{C \sigma}+\Delta A_{C \sigma}\right) x(t)+\left(A_{d \sigma}^{h}+\Delta A_{d \sigma}^{h}\right) x(t-d)+B_{1 \sigma} \omega(t) \\
z(t)=C_{C \sigma} x(t) \\
x(t)=\varphi(t), \forall t \in[-d, 0]
\end{array}\right.
$$

where $\quad \Delta A_{c \sigma}=\Delta A_{\sigma}^{h}+\Delta B_{\sigma}^{h} K_{\sigma}$

Consider switched parameter dependent Lyapunov-like function

$$
V\left(x_{t}\right)=x^{T}(t)\left(\sum_{i=1}^{N} \alpha_{i}(t) P_{i}\right) x(t)+\int_{t-d}^{t} x^{T}(\tau) x(\tau) d \tau
$$

where $x_{t}=x(t+\theta), \theta \in[-d, 0]$ and $P_{i}(i \in \bar{\square})$ are symmetrical positive definite matrices.

Then for any $t \in\left[t_{m}, t_{m+1}\right) \subset \Omega_{r_{m}}$, the time-derivative of Lyapunov-like function (19) along with the trajectory of the closed-loop dynamic system (18) is given by 


$$
\begin{aligned}
& V \&\left(x_{t}\right)=x^{T}(t) P_{r_{m}} x(t)+x^{T}(t) P_{r_{m}} \&(t)+x^{T}(t) x(t)-x^{T}(t-d) x(t-d) \\
& =x^{T}(t)\left[A_{C r_{m}}^{T} P_{r_{m}}+P_{r_{m}} A_{C r_{m}}+\Delta A_{C r_{m}}^{T} P_{r_{m}}+P_{r_{m}} \Delta A_{C r_{m}}+I\right] x(t)+x^{T}(t-d)\left(A_{d r_{m}}^{h}\right)^{T} P_{r_{m}} x(t)+x^{T}(t) P_{r_{m}} A_{d r_{m}}^{h} x(t-d) \\
& +x^{T}(t-d)\left(\Delta A_{d r_{m}}^{h}\right)^{T} P_{r_{m}} x(t)+x^{T}(t) P_{r_{m}} \Delta A_{d r_{m}}^{h} x(t-d)+\omega^{T} B_{1 r_{m}}^{T} P_{r_{m}} x(t)+x^{T}(t) P_{r_{m}} B_{1 r_{m}} \omega-x^{T}(t-d) x(t-d) \\
& \leq x^{T}(t)\left[A_{C r_{m}}^{T} P_{r_{m}}+P_{r_{m}} A_{c_{r_{m}}}+\frac{1}{2} \Delta A_{C r_{m}}^{T} \Delta A_{C_{r_{m}}}+2 P_{r_{m}}^{2}+I\right] x(t)+x^{T}(t-d)\left(A_{d r_{m}}^{h}\right)^{T} P_{r_{m}} x(t) \\
& +x^{T}(t) P_{r_{m}} A_{d r_{m}}^{h} x(t-d)+x^{T}(t-d)\left(\Delta A_{d r_{m}}^{h}\right)^{T} \Delta A_{d r_{m}}^{h} x(t-d)+x^{T}(t) P_{r_{m}}^{2} x(t) \\
& +\gamma^{2} \omega^{T} \omega+\gamma^{-2} x^{T}(t) P_{r_{m}} B_{1 r_{m}} B_{1 r_{m}}^{T} P_{r_{m}} x(t)-x^{T}(t-d) x(t-d)-z^{T}(t) z(t)+x^{T}(t) C_{C r_{m}}^{T} C_{C r_{m}} x(t)
\end{aligned}
$$

By means of (11), we have

$$
\begin{aligned}
\Delta A_{C r_{m}}^{T} \Delta A_{C_{r_{m}}} & =\left(\Delta A_{r_{m}}^{h}(\rho)+\Delta B_{r_{m}}^{h}(\rho) K_{r_{m}}\right)^{T}\left(\Delta A_{r_{m}}^{h}(\rho)+\Delta B_{r_{m}}^{h}(\rho) K_{r_{m}}\right) \\
& \leq 2\left(\Delta A_{r_{m}}^{h}(\rho)\right)^{T} \Delta A_{r_{m}}^{h}(\rho)+2\left(\Delta B_{r_{m}}^{h}(\rho) K_{r_{m}}\right)^{T}\left(\Delta B_{r_{m}}^{h}(\rho) K_{r_{m}}\right) \leq 2 \gamma_{A}^{2} I+2 \gamma_{B}^{2}\left(K_{r_{m}}\right)^{T} K_{r_{m}}
\end{aligned}
$$

and

Consequently,

$$
\left(\Delta A_{d r_{m}}^{h}\right)^{T} \Delta A_{d r_{m}}^{h} \leq \gamma_{A d}^{2} I
$$

$$
\begin{aligned}
& z^{T}(t) z(t)-\gamma^{2} \omega^{T} \omega+v^{\&} \psi\left(x_{t}\right) \\
& \leq x^{T}(t)\left[A_{C r_{m}}^{T} P_{r_{m}}+P_{r_{m}} A_{C r_{m}}+\left(\gamma_{A}^{2}+1\right) I+\gamma_{B}^{2} K_{r_{m}}^{T} K_{r_{m}}+3 P_{r_{m}}^{2}+\gamma^{-2} P_{r_{m}} B_{1 r_{m}} B_{1 r_{m}}^{T} P_{r_{m}}+C_{C r_{m}}^{T} C_{C r_{m}}\right] x(t) \\
& +x^{T}(t-d)\left(A_{d r_{m}}^{h}\right)^{T} P_{r_{m}} x(t)+x^{T}(t) P_{r_{m}} A_{d r_{m}}^{h} x(t-d)+\left(\gamma_{A_{d}}^{2}-1\right) x^{T}(t-d) x(t-d)
\end{aligned}
$$

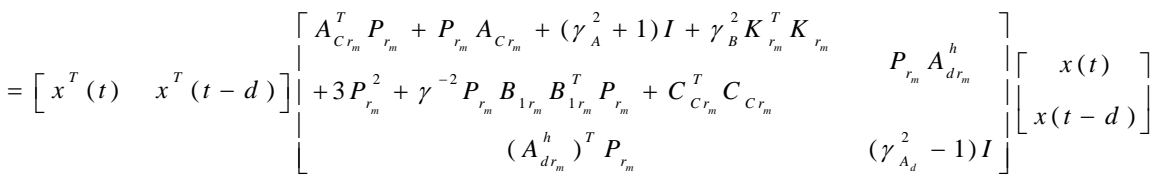

Assume $x(t)=0, \forall t \in[-d, 0]$ and introduce the performance

$$
J=\int_{0}^{T}\left(z^{T}(t) z(t)-\gamma^{2} \omega^{T}(t) \omega(t)\right) d t
$$

Let

$$
\left\{\left(t_{m}, r_{m}\right) \mid r_{m} \in \text { 雍; } m=1,2, \quad, s ; 0=t_{1} \leq t_{2} \leq \mathrm{L} \leq t_{s} \leq T\right\}
$$

be switching sequence in the interval $[0, T]$ that is generated by the switching strategy (14). Noting that $x\left(t_{1}\right)=0$ and $x\left(t_{1}-d\right)=0$, then for every $\omega \in L_{2}[0, T]$.

$$
\begin{aligned}
& J=\sum_{m=1}^{s-1} \int_{t_{m}}^{t_{m+1}}\left(z^{T} z-\gamma^{2} \omega^{T} \omega+V\left(x_{t}\right)\right) d t+\int_{t_{s}}^{T}\left(z^{T} z-\gamma^{2} \omega^{T} \omega+V \&\left(x_{t}\right)\right) d t-V\left(x_{T}\right) \\
& \leq \sum_{m=1}^{s-1} \int_{t_{m}}^{t_{m+1}}\left(z^{T} z-\gamma^{2} \omega^{T} \omega+V\left(x_{t}\right)\right) d t+\int_{t_{s}}^{T}\left(z^{T} z-\gamma^{2} \omega^{T} \omega+\nu \&\left(x_{t}\right)\right) d t
\end{aligned}
$$

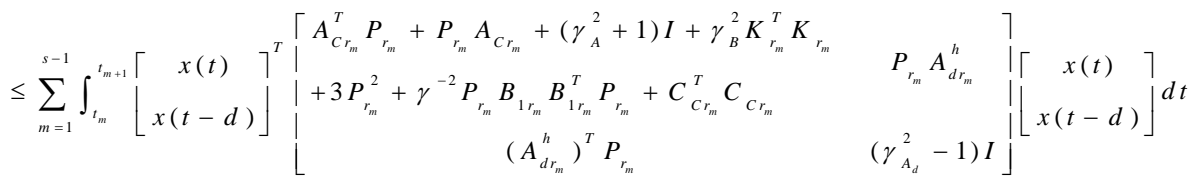

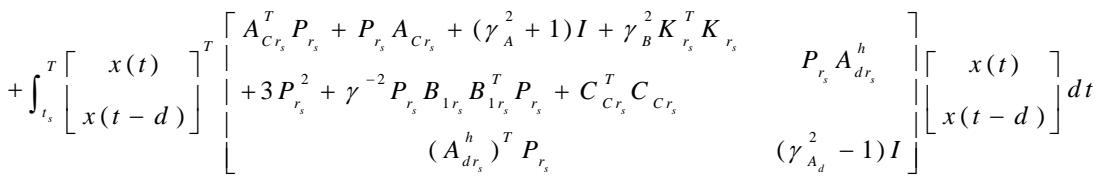

By virtue of the matrix inequality (17), it follows that $J<0$. That is to say

$$
\int_{0}^{T} z^{T}(t) z(t) d t<\gamma^{2} \int_{0}^{T} \omega^{T}(t) \omega(t) d t, \quad \forall \omega \in L_{2}[0, T] .
$$

Now, we prove that the switched system (18) with $\omega=0$ is asymptotically stable.

Let

$$
\left\{\left(t_{m}, r_{m}\right) \mid r_{m} \in \text { 쩎; } m=1,2, \quad ; 0=t_{1} \leq t_{2} \leq \mathrm{L}\right\}
$$

be switching sequence in the interval $[0, \infty)$ that is generated by the switching strategy (14).

For any $t \in\left[t_{m}, t_{m+1}\right) \subset \Omega_{r_{m}}$, the derivative of Lyapunov-like function (19) along with the trajectory of the switched system (18) with $\omega=0$ is given by 


$$
\begin{aligned}
& V \&(x)=x_{t}^{T}(t) P_{r_{m}} x(t)+x^{T}(t) P_{r_{m}} \text { x } \&(t)+x^{T}(t) x(t)-x^{T}(t-d) x(t-d) \\
& =x^{T}(t)\left[A_{C r_{m}}^{T} P_{r_{m}}+P_{r_{m}} A_{C r_{m}}+\Delta A_{C r_{m}}^{T} P_{r_{m}}+P_{r_{m}} \Delta A_{C r_{m}}+I\right] x(t)+x^{T}(t-d)\left(A_{d r_{m}}^{h}\right)^{T} P_{r_{m}} x(t) \\
& +x^{T}(t) P_{r_{m}} A_{d r_{m}}^{h} x(t-d)+x^{T}(t-d)\left(\Delta A_{d r_{m}}^{h}\right)^{T} P_{r_{m}} x(t)+x^{T}(t) P_{r_{m}} \Delta A_{d r_{m}}^{h} x(t-d)-x^{T}(t-d) x(t-d)
\end{aligned}
$$

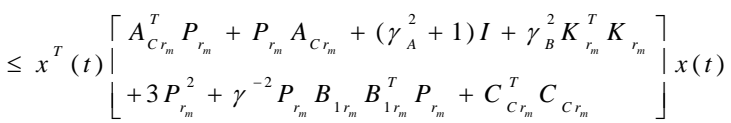

$$
\begin{aligned}
& +x^{T}(t-d)\left(A_{d r_{m}}^{h}\right)^{T} P_{r_{m}} x(t)+x^{T}(t) P_{r_{m}} A_{d r_{m}}^{h} x(t-d)+\left(\gamma_{A_{d}}^{2}-1\right) x^{T}(t-d) x(t-d) \\
& \left.=\left[\begin{array}{c}
x(t) \\
x(t-d)
\end{array}\right\rceil^{T} \mid \begin{array}{cc}
A_{C r_{m}}^{T} P_{r_{m}}+P_{r_{m}} A_{C_{r_{m}}}+\left(\gamma_{A}^{2}+1\right) I+\gamma_{B}^{2} K_{r_{m}}^{T} K_{r_{m}} & P_{r_{m}} A_{d r_{m}}^{h} \backslash\left[\begin{array}{c}
x(t) \\
+3 P_{r_{m}}^{2}+\gamma^{-2} P_{r_{m}} B_{1 r_{m}} B_{1 r_{m}}^{T} P_{r_{m}}+C_{C r_{m}}^{T} C_{C_{r_{m}}}
\end{array}\right. \\
\left(A_{d r_{m}}^{h}\right)^{T} P_{r_{m}} & \left(\gamma_{A_{d}}^{2}-1\right) I
\end{array}\right]
\end{aligned}
$$

By means of (17), for any $t \in\left[t_{m}, t_{m+1}\right) \subset \Omega_{r_{m}}$, the time derivative of Lyapunov-like functions (19) alone with the trajectory of the switched system (18) with $\omega=0$ is less than zero.

Without of generally, suppose $\sigma\left(t_{m+1}^{+}\right)=r_{m+1}$. Then by Lyapunov-like functions (19), switching strategy (14) and $P_{i}^{-1}=X_{i}, i=1,2, \cdots, N$.

$$
V\left(t_{m+1}^{+}\right)-V\left(t_{m+1}^{-}\right)=x^{T}\left(t_{m+1}\right)\left(P_{r_{m+1}}-P_{r_{m}}\right) x\left(t_{m+1}\right)=x^{T}\left(t_{m+1}\right)\left(X_{r_{m+1}}^{-1}-X_{r_{m}}^{-1}\right) x\left(t_{m+1}\right)<0
$$

Hence under the action of switching strategy $\sigma(t)$ and associated state feedback sub-controllers $u=K_{i} x(t), i \in \bar{\square}$, the state delay switched linear system (3) is asymptotically stabilization with $H_{\infty}$ disturbance attenuation level $\gamma$ via switched state feedback.

\section{Robust $H_{\infty}$ Control via dynamic Output Feedback}

In this section, the robust $H_{\infty}$ problem of state delay switched linear system (2) is investigated via switched output feedback. Before the design of switched stabilization controller, we firstly show how the system (2) can be expressed as a switched polytopic system with an additive norm bounded uncertainty. Such a formulation makes the robust stabilization problem tractable.

By Lemma 3.1, the following lemma is obvious.

Lemma 4.1: The state delay switched linear system (2) subject to exponential uncertainties can be expressed as:

$$
\left\{\begin{aligned}
\&(t)= & \left(A_{\sigma}^{h}(\rho(t))+\Delta A_{\sigma}^{h}(\rho(t))\right) x(t)+\left(A_{d \sigma}^{h}(\rho(t))+\Delta A_{d \sigma}^{h}(\rho(t))\right) x(t-d) \\
& +\left(B_{\sigma}^{h}(\rho(t))+\Delta B_{\sigma}^{h}(\rho(t))\right) u(t)+B_{1 \sigma} \omega(t) \\
z(t)= & C_{1 \sigma} x(t)+D_{\sigma} u(t) \\
y(t)= & C_{2 \sigma} x(t) \\
x(t)= & \varphi(t), \forall t \in[-d, 0]
\end{aligned}\right.
$$

where $A_{\sigma}^{h}(\rho(t)), A_{d \sigma}^{h}(\rho(t))$ and $B_{\sigma}^{h}(\rho(t))$ are described as (4)-(6) and (8)-(9); the remainders of the Taylor series approximation $\Delta A_{\sigma}^{h}(\rho(t)), \Delta A_{d \sigma}^{h}(\rho(t))$ and $\Delta B_{\sigma}^{h}(\rho(t))$ are given by the functions (10)-(12). The description of parameters $\mu_{j}(\rho(t))$ is given by (7) and Lemma 2.1 .

In view of the Lemma 4.1, switched dynamic model (20) is actually a switched polytopic system subject to norm bounded uncertainty. Furthermore, such a switched model can be used to represent the state delay switched linear system (2). Therefore, stabilizing switched dynamic model (20) is equivalent to doing the system (2). 
Our goal is, for any given $\gamma>0$, to find $\mathrm{N}$ switched dynamic output feedback subcontrollers $u=k_{i}(s) y, i=1,2, \cdots N$ such that the resulting closed-loop system (20) satisfies:

a) With zero disturbance input condition $\omega \equiv 0$, it is asymptotically stable for all admissible uncertainties.

b) With initial condition $x(t)=0, \forall t \in[-d, 0], \int_{0}^{T} z^{T}(t) z(t) d t<\gamma^{2} \int_{0}^{T} \omega^{T}(t) \omega(t) d t$ for all nonzero $\omega \in L_{2}[0, T](0 \leq T<\infty)$ and all admissible uncertainties.

For system (20), we are interested in constructing the form of the switched dynamic output-feedback controller as follows:

$$
\left\{\begin{array}{l}
\xi(t)=\hat{A}_{\sigma(t)} \xi(t)+\hat{B}_{\sigma(t)} y(t) \\
u(t)=\hat{C}_{\sigma(t)} \xi(t)+\hat{D}_{\sigma(t)} y(t)
\end{array}\right.
$$

where $\xi \in i^{n}$.

In this case, the resulting closed-loop system (20) with switched dynamic outputfeedback controller (21) is given by

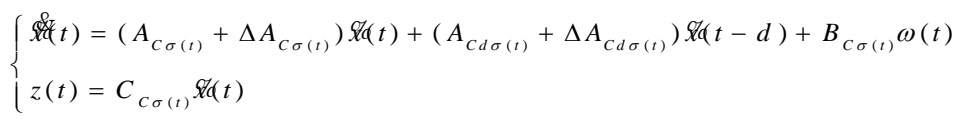

where

$$
\begin{aligned}
& 9_{0}^{T}=\left(x^{T}, \xi^{T}\right), A_{C \sigma(t)}=A_{\sigma(t)}^{0}+B_{\sigma(t)}^{0} K_{\sigma(t)} C_{2 \sigma(t)}^{0}, \Delta A_{C \sigma(t)}=\Delta A_{\sigma(t)}^{0}+\Delta B_{\sigma(t)}^{0} K_{\sigma(t)} C_{2 \sigma(t)}^{0}, \\
& C_{C \sigma(t)}=C_{1 \sigma(t)}^{0}+D_{\sigma(t)}^{0} K_{\sigma(t)} C_{2 \sigma(t)}^{0}, C_{1 \sigma(t)}^{0}=\left[\begin{array}{ll}
C_{1 \sigma(t)} & 0
\end{array}\right], B_{C \sigma(t)}=B_{1 \sigma(t)}^{0}=\left\lceil\begin{array}{c}
B_{1 \sigma(t)} \\
0
\end{array}\right], \\
& A_{C d \sigma(t)}=\left[\begin{array}{cc}
A_{d \sigma(t)}^{h} & 0 \\
0 & 0
\end{array}\right], \Delta A_{C d \sigma(t)}=\left[\begin{array}{cc}
\Delta A_{d \sigma(t)}^{h} & 0 \\
0 & 0
\end{array}\right], A_{\sigma(t)}^{0}=\left[\begin{array}{cc}
A_{\sigma(t)}^{h} & 0 \\
0 & 0
\end{array}\right], \\
& \Delta A_{\sigma(t)}^{0}=\left[\begin{array}{cc}
\Delta A_{\sigma(t)}^{h} & 0 \\
0 & 0
\end{array}\right], D_{\sigma(t)}^{0}=\left[\begin{array}{cc}
D_{\sigma(t)} & 0
\end{array}\right], B_{\sigma(t)}^{0}=\left[\begin{array}{cc}
B_{\sigma(t)}^{h} & 0 \\
0 & I
\end{array}\right], \\
& \Delta B_{\sigma(t)}^{0}=\left[\begin{array}{cc}
\Delta B_{\sigma(t)}^{h} & 0 \\
0 & 0
\end{array}\right], C_{2 \sigma(t)}^{0}=\left[\begin{array}{cc}
C_{2 \sigma(t)} & 0 \\
0 & I
\end{array}\right], K_{\sigma(t)}=\left[\begin{array}{ll}
\hat{D}_{\sigma(t)} & \hat{C}_{\sigma(t)} \\
\hat{B}_{\sigma(t)} & \hat{A}_{\sigma(t)}
\end{array}\right] .
\end{aligned}
$$

Next, we give the sufficient conditions for existence of the switching strategy $\sigma(t)$ and associated dynamic output feedback sub-controllers (21) such that the resulting closedloop system (22) is stabilizable with $H_{\infty}$ disturbance attenuation level $\gamma$.

Theorem 4.1: Given any constant $\gamma>0$, the uncertain state delay switched linear system (20) is asymptotically stabilization with $H_{\infty}$ disturbance attenuation $\gamma$ via switched dynamic output feedback if there exist symmetrically positive definite matrices $X_{C i}$ with $i=1,2, \mathrm{~L}, N$ such that the following matrix inequality is satisfied for any $i=1,2, \mathrm{~L}, N$.

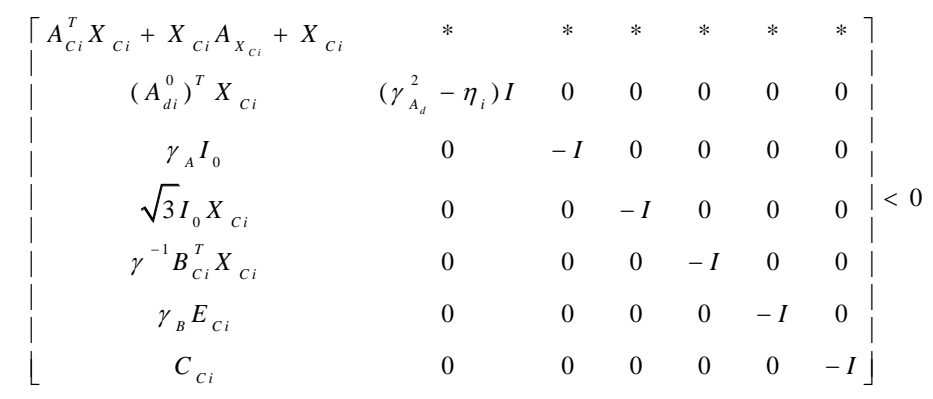

where $A_{d i}^{0}=\left[\begin{array}{c}A_{d i}^{h} \\ 0\end{array}\right], 0<\eta_{i} \leq \lambda_{\text {m in }}\left(X_{C i}\right), I_{0}=\left[\begin{array}{ll}I & 0\end{array}\right], I_{C}=I_{0}^{T} I_{0}=\left[\begin{array}{ll}I & 0 \\ 0 & 0\end{array}\right], E_{C i}=I_{0} K_{i} C_{2 i}^{0}$.

In this case, the dynamic output feedback controller gain matrix 


$$
K_{i}=\left[\begin{array}{ll}
\hat{D}_{i} & \hat{C}_{i} \\
\hat{B}_{i} & \hat{A}_{i}
\end{array}\right], \quad i=1,2, \mathrm{~L}, N
$$

The switching strategy $\sigma(t)$ is given by

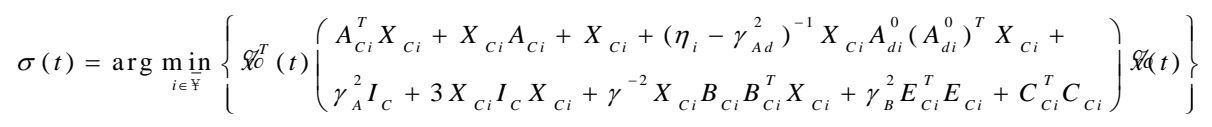

Proof: The matrix inequality (23) is transformed into the following matrix inequality.

$$
\left\{\begin{array}{ccccccc}
A_{C i}^{T} X_{C i}+X_{C i} A_{X_{C i}}+X_{C i} & * & * & * & * & * & * \\
A_{C d i}^{T} X_{C i} & \gamma_{A_{d}}^{2} I_{C}-\eta_{i} I & 0 & 0 & 0 & 0 & 0 \\
\gamma_{A} I_{0} & 0 & -I & 0 & 0 & 0 & 0 \\
\sqrt{3} I_{0} X_{C i} & 0 & 0 & -I & 0 & 0 & 0 \\
\gamma^{-1} B_{C i}^{T} X_{C i} & 0 & 0 & 0 & -I & 0 & 0 \\
\gamma_{B} E_{C i} & 0 & 0 & 0 & 0 & -I & 0 \\
C_{C i} & 0 & 0 & 0 & 0 & 0 & -I
\end{array}\right]
$$

In view of Lemma 2.2, the matrix inequality (26) is equivalent to the following inequality.

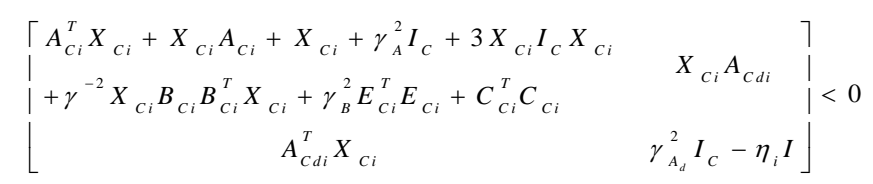

Consider the notations:

$$
\alpha_{i}(t)= \begin{cases}1 & t \in \Omega_{i} \\ 0 & t \notin \Omega_{i}\end{cases}
$$

$\Omega_{i}=\{t \mid$ the $\mathrm{i}$-th subsystem is active at time instant $t\}, i=1,2, \cdots, N$.

Consider switched parameter dependent Lyapunov-like function

$$
V\left(\tilde{x}_{t}\right)=\tilde{x}^{T}(t)\left(\sum_{i=1}^{N} \alpha_{i}(t) P_{i}\right) \tilde{x}(t)+\int_{t-d}^{t} \tilde{x}^{T}(\tau)\left(\sum_{i=1}^{N} \alpha_{i}(t) P_{i}\right) \tilde{x}(\tau) d \tau
$$

where $\tilde{x}_{t}=\tilde{x}(t+\theta), \theta \in[-d, 0]$ and $P_{i}(i \in \bar{\square})$ are symmetrical positive definite matrices.

Then for any $t \in\left[t_{m}, t_{m+1}\right) \subset \Omega_{r_{m}}$, the time-derivative of Lyapunov-like function (28) along with the trajectory of the closed-loop dynamic system (22) is given by

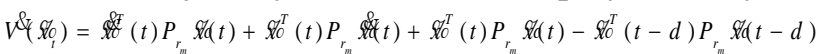

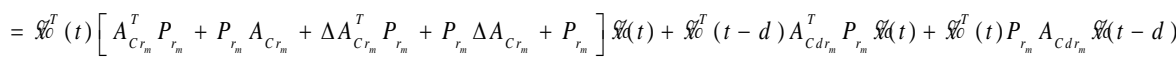

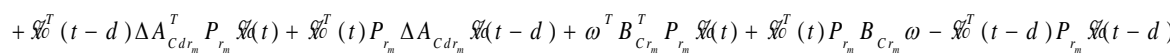

Again in view of (11),

$$
\begin{aligned}
& \Delta A_{C r_{m}}^{T} P_{r_{m}}+P_{r_{m}} \Delta A_{C r_{m}} \leq \frac{1}{2} \Delta A_{C r_{m}}^{T} \Delta A_{C r_{m}}+2 P_{r_{r_{m}}} I_{C} P_{r_{m}} \leq \gamma_{A}^{2} I_{C}+\gamma_{B}^{2} E_{C r_{m}}^{T} E_{C r_{m}}+2 P_{r_{m}} I_{C} P_{r_{m}} \\
& \Delta A_{C d_{m}}^{T} P_{r_{m}}+P_{r_{m}} \Delta A_{C r_{r_{m}}} \leq \Delta A_{C d_{r_{m}}}^{T} \Delta A_{C d_{r_{m}}}+P_{r_{r_{m}}} I_{C} P_{r_{m}} \leq \gamma_{A d}^{2} I_{C}++P_{r_{m}} I_{C} P_{r_{m}}
\end{aligned}
$$

Consequently,

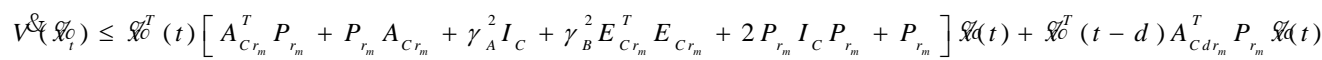

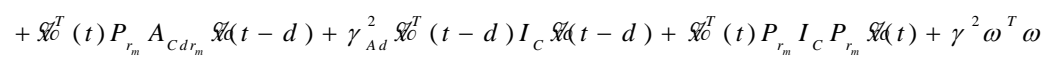

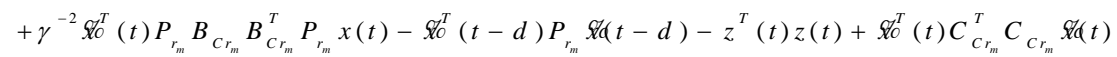

By $P_{r_{m}} \geq \eta_{r_{m}} I>0$, we have 


$$
\begin{aligned}
& \left.z^{T}(t) z(t)-\gamma^{2} \omega^{T} \omega+V L_{t}\right)
\end{aligned}
$$

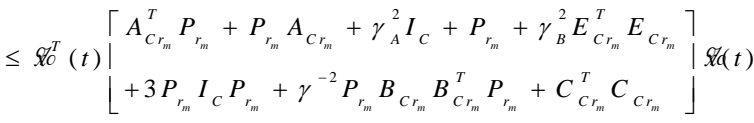

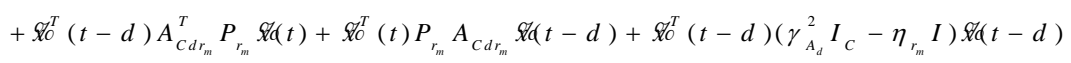

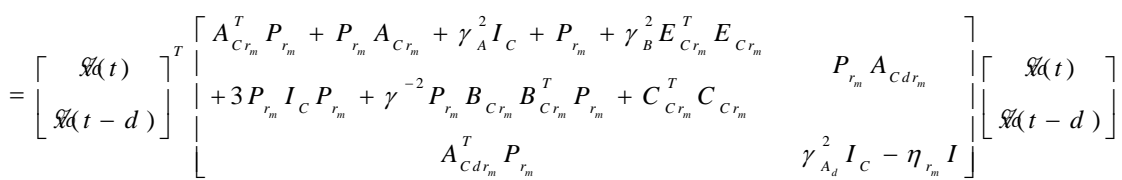

Assume $\tilde{x}(t)=0, \forall t \in[-d, 0]$ and introduce the performance

$$
J=\int_{0}^{T}\left(z^{T}(t) z(t)-\gamma^{2} \omega^{T}(t) \omega(t)\right) d t
$$

Let

$$
\left\{\left(t_{m}, r_{m}\right) \mid r_{m} \in \text { 쩖; } m=1,2, \quad, s ; 0=t_{1} \leq t_{2} \leq \mathrm{L} \leq t_{s} \leq T\right\}
$$

be switching sequence in the interval $[0, T]$ that is generated by the switching strategy (25). Noting that $\tilde{x}\left(t_{1}\right)=0$ and $\tilde{x}\left(t_{1}-d\right)=0$, then for every $\omega \in L_{2}[0, T]$.

$$
\begin{aligned}
& J=\sum_{m=1}^{s-1} \int_{t_{m}}^{t_{m+1}}\left(z^{T} z-\gamma^{2} \omega^{T} \omega+V \psi\left(\bigotimes_{t}\right)\right) d t+\int_{t_{s}}^{T}\left(z^{T} z-\gamma^{2} \omega^{T} \omega+V\left(\phi_{t}\right)\right) d t-V\left(\varnothing_{T}\right) \\
& \leq \sum_{m=1}^{s-1} \int_{t_{m}}^{t_{m+1}}\left(z^{T} z-\gamma^{2} \omega^{T} \omega+V_{t}\left(\phi_{t}\right)\right) d t+\int_{t_{s}}^{T}\left(z^{T} z-\gamma^{2} \omega^{T} \omega+\nu\left(\phi_{t}\right)\right) d t
\end{aligned}
$$

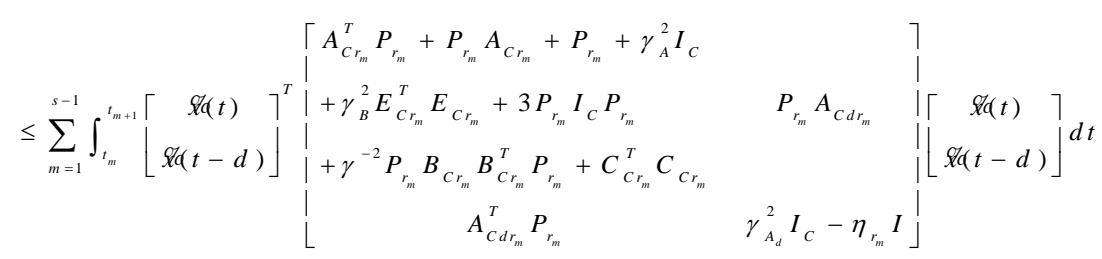

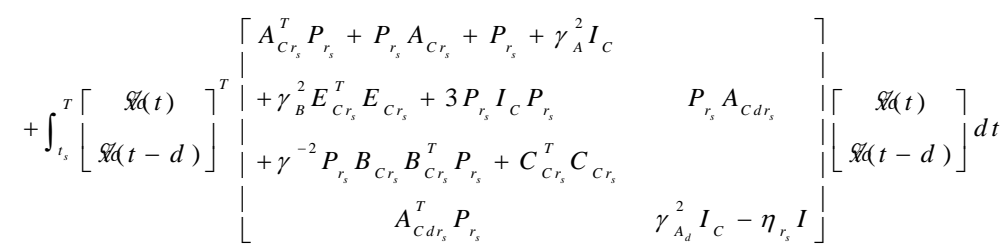

Setting $P_{r_{m}}=X_{C_{r_{m}}}>0$, by virtue of the matrix inequality (27), it follows that $J<0$. That is

$$
\int_{0}^{T} z^{T}(t) z(t) d t<\gamma^{2} \int_{0}^{T} \omega^{T}(t) \omega(t) d t, \quad \forall \omega \in L_{2}[0, T] .
$$

Now, we prove that the switched system (20) with $\omega=0$ is asymptotically stable.

Let $\quad\left\{\left(t_{m}, r_{m}\right) \mid r_{m} \in\right.$ ㅉㅉㄻ; $\left.m=1,2, \quad ; 0=t_{1} \leq t_{2} \leq \mathrm{L}\right\}$ be switching sequence in the interval $[0, \infty)$ that is generated by the switching strategy (25).

For any $t \in\left[t_{m}, t_{m+1}\right) \subset \Omega_{r_{m}}$, the derivative of Lyapunov-like function (28) along with the trajectory of the switched system (22) with $\omega=0$ is given by

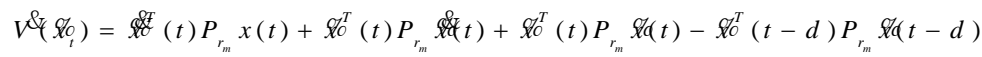

$$
\begin{aligned}
& =80^{T}(t)\left[A_{C r_{m}}^{T} P_{r_{m}}+P_{r_{m}} A_{C r_{m}}+\Delta A_{C r_{m}}^{T} P_{r_{m}}+P_{r_{m}} \Delta A_{C r_{m}}+P_{r_{m}}\right] \text { S } d(t)+80^{T}(t-d) A_{C d r_{m}}^{T} P_{r_{m}} \operatorname{SB}(t)
\end{aligned}
$$

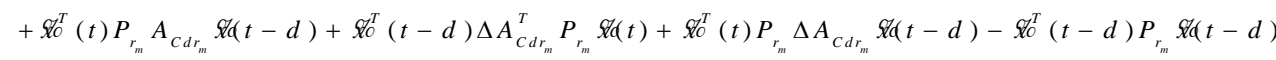

$$
\begin{aligned}
& \leq 80^{T}(t) ! \begin{array}{l}
\left.A_{C r_{m}}^{T} P_{r_{m}}+P_{r_{m}} A_{C r_{m}}+\gamma_{A}^{2} I_{C}+\gamma_{B}^{2} E_{C r_{m}}^{T} E_{C r_{m}}+P_{r_{m}}\right\rceil S q(t) \\
\left.+3 P_{r_{m}} I_{C} P_{r_{m}}+\gamma^{-2} P_{r_{m}} B_{C r_{m}} B_{C r_{m}}^{T} P_{r_{m}}+C_{C r_{m}}^{T} C_{C r_{m}}\right\rfloor
\end{array}
\end{aligned}
$$

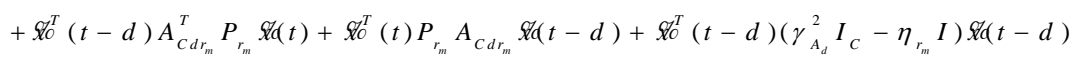




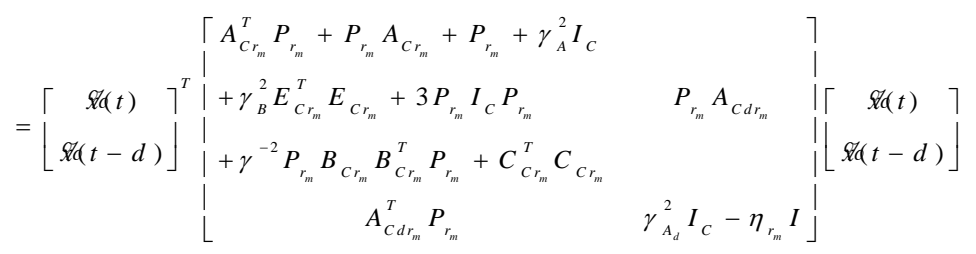

By means of (27), for any $t \in\left[t_{m}, t_{m+1}\right) \subset \Omega_{r_{m}}$, the time derivative of Lyapunov-like functions (28) alone with the trajectory of the switched system (22) with $\omega=0$ is less than zero.

Without of generally, suppose that $\sigma\left(t_{m+1}^{+}\right)=r_{m+1}$. Then by Lyapunov-like functions (28) and switching strategy (25) and $P_{i}=X_{C i}, i=1,2, \cdots, N$

$$
V\left(\tilde{x}_{t_{m+1}^{+}}\right)-V\left(\tilde{x}_{t_{m+1}^{-}}\right)=\tilde{x}^{T}\left(t_{m+1}\right)\left(X_{C r_{m+1}}-X_{C r_{m}}\right) \tilde{x}\left(t_{m+1}\right)+\int_{t_{m+1}-d}^{t_{m+1}} \tilde{x}^{T}(\tau)\left(X_{C r_{m+1}}-X_{C r_{m}}\right) \tilde{x}(\tau) d(\tau)<0
$$

Hence under the action of switching strategy $\sigma(t)$ and associated dynamic output feedback sub-controllers (21), the state delay switched linear system (2) is asymptotically stabilization with $H_{\infty}$ disturbance attenuation level $\gamma$ via switched dynamic output feedback.

However, the matrix inequality (23) is not linear matrix inequality. Our goal in the following section, for the non-linear matrix inequality (23), is transformed into linear matrix inequalities by the so-called eliminate element method (a kind of classical technique in studying the $H_{\infty}$ control problem of a non-switched linear system).

The matrix inequality (23) is transformed into the following matrix inequality.

$$
H_{X_{C i}}+M_{X_{C i}}^{T} K_{i} Q_{i}+Q_{i}^{T} K_{i}^{T} M_{X_{C i}}<0
$$

where

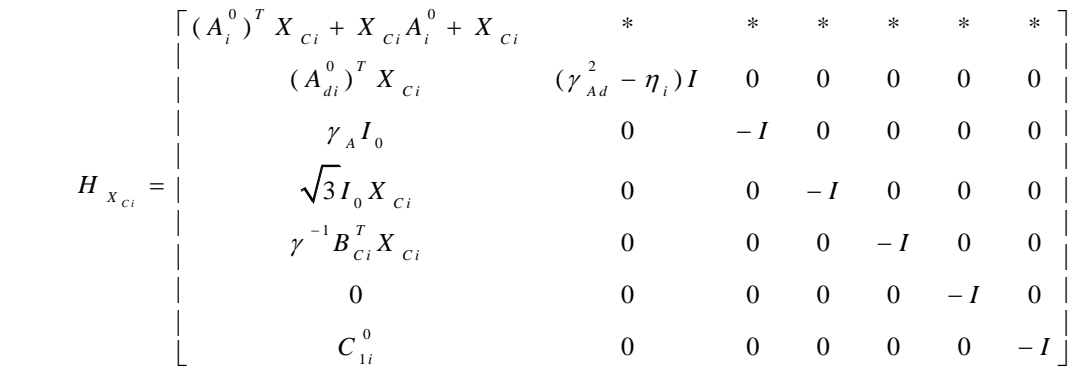

$$
\begin{aligned}
& M_{X_{c i}}=\left[\begin{array}{lllllll}
\left(B_{i}^{0}\right)^{T} X_{C i} & 0 & 0 & 0 & 0 & \gamma_{B} I_{0}^{T} & \left(D_{i}^{0}\right)^{T}
\end{array}\right], Q_{i}=\left[\begin{array}{lllllll}
C_{2 i}^{0} & 0 & 0 & 0 & 0 & 0 & 0
\end{array}\right] .
\end{aligned}
$$

By Lemma 2.3, the matrix inequality (29) is equivalent to the following inequalities.

$$
M_{X_{C i}}^{\perp T} H_{X_{C i}} M_{X_{C i}}^{\perp}<0 \text { and } Q_{i}^{\perp T} H_{X_{C i}} Q_{i}^{\perp}<0 .
$$

For $M_{X_{c i}}^{\perp T} H_{X_{c i}}^{\perp T} M_{X_{c i}}^{\perp}<0$, setting $S_{i}=\operatorname{diag}\left\{X_{c i}, I, I, I, I, I, I\right\}$, then $M_{X_{c i}}=M_{i} S_{i}$, where $M_{i}=\left[\begin{array}{llllllll}\left(B_{2 i}^{0}\right)^{T} & 0 & 0 & 0 & 0 & \gamma_{B} I_{0}^{T} & \left(D_{i}^{0}\right)^{T}\end{array}\right]$. So we have $M_{x_{C 1}}^{\perp}=S_{i}^{-1} M_{i}^{\perp}$.

The matrix inequality $M_{X_{c i}}^{\perp T} H_{X_{c i}} M_{X_{c i}}^{\perp}<0$ is transformed into the following inequality:

where

$$
M_{i}^{\perp T} T_{X_{C i}} M_{i}^{\perp}<0
$$




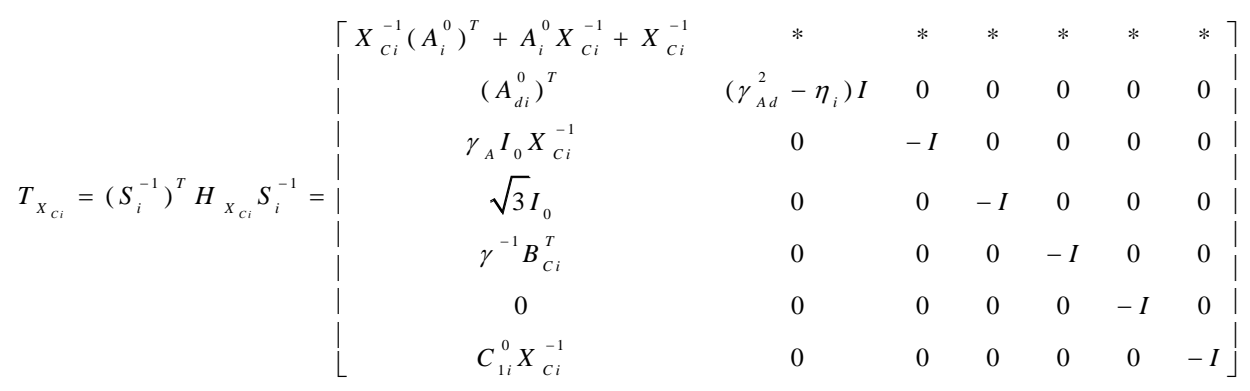

On the one hand in view of Lemma 2.4 and the definition of $A_{i}^{0}, A_{d i}^{0}, B_{C i}$ and $C_{1 i}^{0}$, the above matrix is transformed into the following matrix.

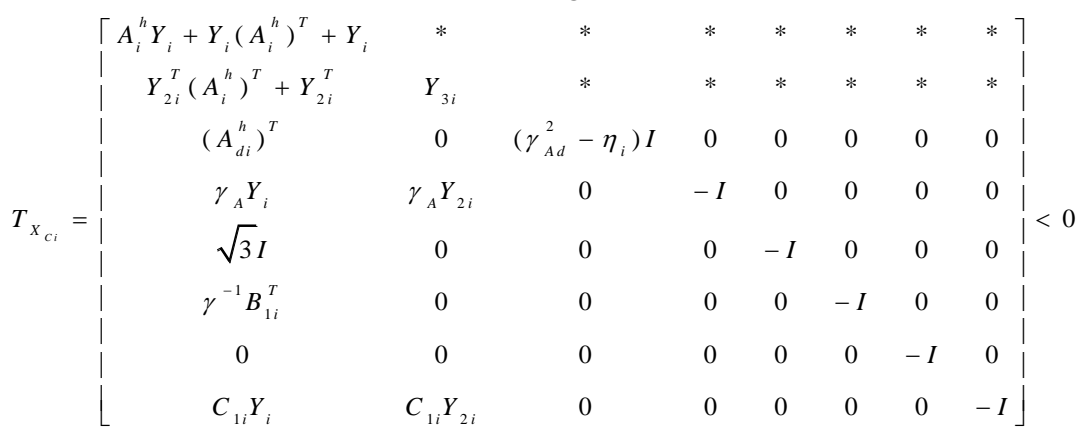

On the other hand by the definition of $B_{2 i}^{0}, D_{i}^{0}$ and $I_{0}$, the matrix $M_{i}$ is transformed into the following matrix.

$$
M_{i}=\left[\begin{array}{cccccccc}
\left(B_{i}^{h}\right)^{T} & 0 & 0 & 0 & 0 & 0 & \gamma_{B} I & D_{i}^{T} \\
0 & I & 0 & 0 & 0 & 0 & 0 & 0
\end{array}\right]
$$

Then, we have

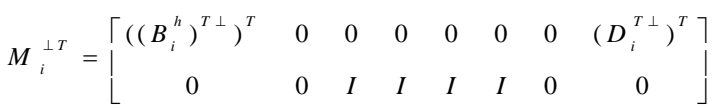

Hence, the matrix inequality $M_{i}^{\perp T} T_{X_{c i}} M_{i}^{\perp}<0$ is transformed into the following inequality.

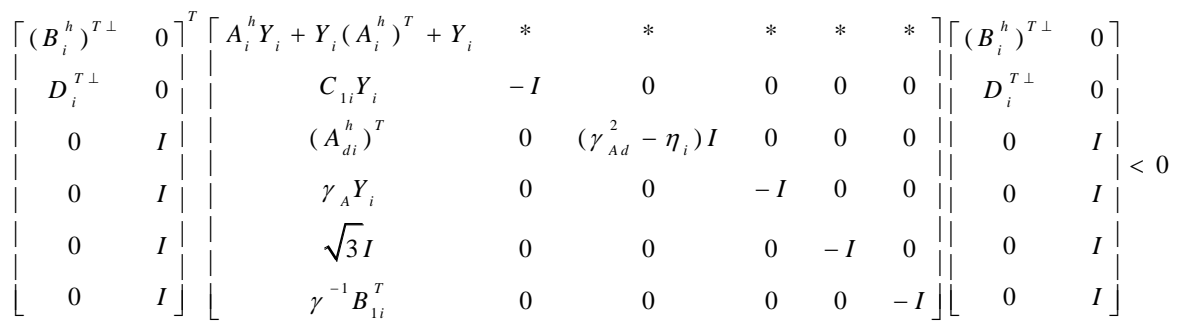

According to the above same method, the matrix inequality $Q_{i}^{\perp T} H_{X_{c i}} Q_{i}^{\perp}<0$ is transformed into the following inequality.

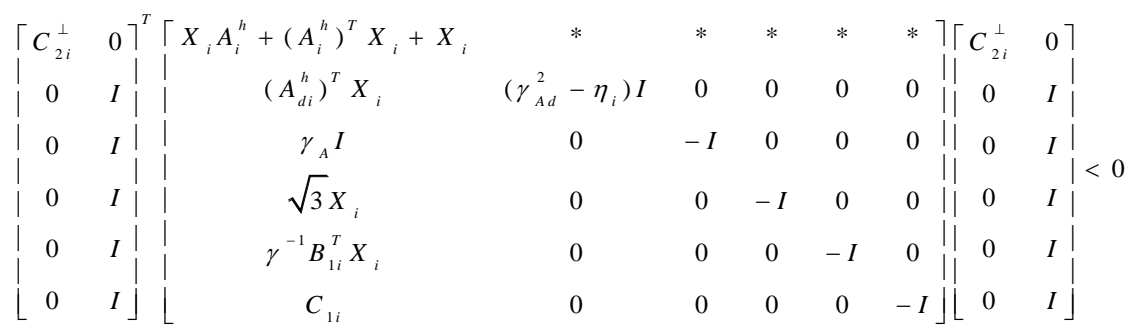

In summary, for disturbance attenuation performance of system (2), we have the following result.

Theorem 4.2 Given any constant $\gamma>0$, the uncertain state delay switched linear 
system (2) is said to be asymptotically stabilizable with $H_{\infty}$ disturbance attenuation level $\gamma$ via switched dynamic output feedback, if there exist symmetrically positive definite matrices $X_{i}$ and $Y_{i}$ with $i \in \bar{\square}$ such that the following linear matrix inequality, linear matrix inequalities (21) and (22) are satisfied for all $i \in \bar{\square}$.

$$
\left[\begin{array}{cc}
X_{i} & I \\
I & Y_{i}
\end{array}\right] \geq 0
$$

The switching strategy $\sigma(t)$ is given by (25);

In this case, the dynamic output feedback controller gain matrix

$$
K_{i}=\left[\begin{array}{ll}
\hat{D}_{i} & \hat{C}_{i} \\
\hat{B}_{i} & \hat{A}_{i}
\end{array}\right], \quad i=1,2, \mathrm{~L}, N
$$

can be solved by using the following algorithm.

Step 1: To solve the matrices $X_{i}$ and $Y_{i}$ by using the conditions (31)-(33).

Step 2: We first solve $X_{2 i} \in i^{n \times n_{k}}$ via the matrix equality $X_{2 i} X_{2 i}^{T}=X_{i}-Y_{i}^{-1}$, and then construct the matrix $X_{c i}=\left[\begin{array}{cc}X_{i} & X_{2 i} \\ X_{2 i}^{T} & I\end{array}\right]>0$, where $n_{k}=\operatorname{rank}\left(X_{i}-Y_{i}^{-1}\right)$.

Step 3: To solve $K_{i}$ via $H_{X_{c i}}+M_{X_{c i}}^{T} K_{i} Q_{i}+Q_{i}^{T} K_{i}^{T} M_{X_{c i}}<0$.

Proof: The proof of Theorem 4.2 is obvious.

\section{Numerical example}

In this section, we use two examples to show the benefits effective of our results.

Example 1: Consider the state delay switched linear system (3) with $N=2$, $\sigma(t):[0, \infty) \rightarrow\{1,2\}$ and

$$
\begin{aligned}
& M_{1}=\left[\begin{array}{ll}
-3 & -2 \\
1 & -1
\end{array}\right], M_{2}=\left[\begin{array}{cc}
2 & -7 \\
0 & 1
\end{array}\right], R_{1}=\left[\begin{array}{cc}
-1 & -1 \\
2 & 1
\end{array}\right], R_{2}=\left[\begin{array}{cc}
-1 & 0 \\
4 & -1
\end{array}\right], \\
& N_{1}=\left[\begin{array}{ll}
-18 & -5 \\
-4 & 11
\end{array}\right], N_{2}=\left[\begin{array}{cc}
-5 & 6 \\
-15 & 0
\end{array}\right], B_{11}=\left[\begin{array}{cc}
-1 & 2 \\
1 & -1
\end{array}\right], B_{12}=\left[\begin{array}{cc}
-2 & 1 \\
1 & -1
\end{array}\right], \\
& C_{1}=\left[\begin{array}{ll}
3 & -5 \\
1 & -3
\end{array}\right], C_{2}=\left[\begin{array}{ll}
-6 & 2 \\
-1 & 1
\end{array}\right], D_{1}=\left[\begin{array}{cc}
-3 & 1 \\
1 & 1
\end{array}\right], D_{2}=\left[\begin{array}{ll}
-3 & 2 \\
-1 & 2
\end{array}\right] .
\end{aligned}
$$

Set $\underline{\rho}=0.1, \bar{\rho}=0.5, \rho_{1}=0.3, \rho_{2}=0.2, h=3$ and the disturbance attenuation level $\gamma=0.9$. Then, by theorem 3.1, we have

$$
\begin{array}{ll}
X_{1}=\left[\begin{array}{ll}
1.8793 & 0.5732 \\
0.5732 & 0.2932
\end{array}\right], & Y_{1}=\left[\begin{array}{ll}
1.6772 & -0.1442 \\
1.5326 & -2.3549
\end{array}\right] ; \\
X_{2}=\left[\begin{array}{cc}
0.4778 & -0.3619 \\
-0.3619 & 6.5929
\end{array}\right], & Y_{2}=\left[\begin{array}{ll}
-3.2924 & 7.2986 \\
-2.5587 & 1.6661
\end{array}\right]
\end{array}
$$

and by (14), the gain matrices of the state feedback sub-controller are given by

$$
K_{1}=\left[\begin{array}{ll}
2.5817 & -5.5387 \\
8.0861 & -23.8392
\end{array}\right], \quad K_{2}=\left[\begin{array}{cc}
-6.3148 & 0.7604 \\
-5.3877 & -0.0430
\end{array}\right]
$$

Example 2:Consider the state delay switched linear system (20) with $N=2$, $\sigma(t):[0, \infty) \rightarrow\{1,2\}$ and

$$
\begin{aligned}
& M_{1}=\left[\begin{array}{cc}
-1 & -15 \\
0.05 & 1.9
\end{array}\right], M_{2}=\left[\begin{array}{cc}
-1.54 & -99 \\
0 & 1
\end{array}\right], R_{1}=\left[\begin{array}{ll}
0.2 & -0.5 \\
0.1 & -0.3
\end{array}\right], R_{2}=\left[\begin{array}{cc}
2 & 0.5 \\
0.5 & -0.1
\end{array}\right], \\
& N_{1}=\left[\begin{array}{cc}
15 & -2 \\
1 & 7
\end{array}\right], N_{2}=\left[\begin{array}{cc}
-2 & 6 \\
-3 & 1
\end{array}\right], B_{11}=\left[\begin{array}{cc}
-2 & 2 \\
4 & -2
\end{array}\right], B_{12}=\left[\begin{array}{cc}
-4 & 0 \\
2 & 0
\end{array}\right], C_{11}=\left[\begin{array}{cc}
-7 & 1 \\
2 & 0.5
\end{array}\right],
\end{aligned}
$$




$$
C_{12}=\left[\begin{array}{cc}
-4 & -1 \\
-3 & 1
\end{array}\right], C_{21}=\left[\begin{array}{ll}
1 & 0 \\
1 & 0
\end{array}\right], C_{22}=\left[\begin{array}{cc}
-1 & 0 \\
3 & 0
\end{array}\right], D_{1}=\left[\begin{array}{cc}
-2 & 2 \\
0 & 0
\end{array}\right], D_{2}=\left[\begin{array}{cc}
-3 & -2 \\
0 & 0
\end{array}\right] .
$$

Set $\rho=0.1, \bar{\rho}=0.5, \rho_{1}=0.3, \rho_{2}=0.2, \eta_{1}=0.01, \eta_{2}=0.05, h=3$, and the disturbance attenuation level $\gamma=0.9$.

Then by theorem 4.2 , we have

$$
\begin{array}{rlr}
X_{1}=\left[\begin{array}{ll}
11.8487 & 1.4274 \\
1.4274 & 0.7331
\end{array}\right], & Y_{1}=\left[\begin{array}{cc}
4.2763 & -1.4503 \\
-1.4503 & 12.2084
\end{array}\right], \\
X_{2}=\left[\begin{array}{ll}
3.5762 & 0.1934 \\
0.1934 & 0.7214
\end{array}\right], & Y_{2}=\left[\begin{array}{cc}
3.5608 & -0.0198 \\
-0.0198 & 3.8525
\end{array}\right] .
\end{array}
$$

Again by Theorem 4.2, system (20) with $N=2$ satisfies robust $H_{\infty}$ performance with the disturbance attenuation level $\gamma=0.9$ via dynamic output feedback controllers and a switching strategy, where the dynamic output feedback controllers are given by

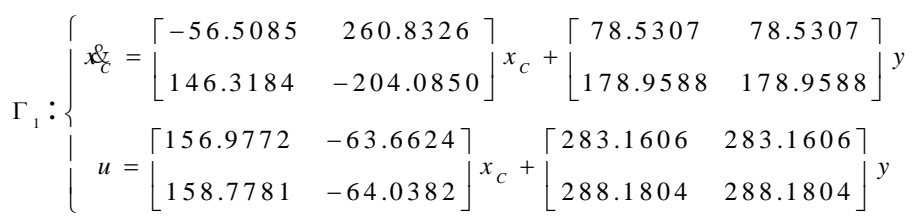

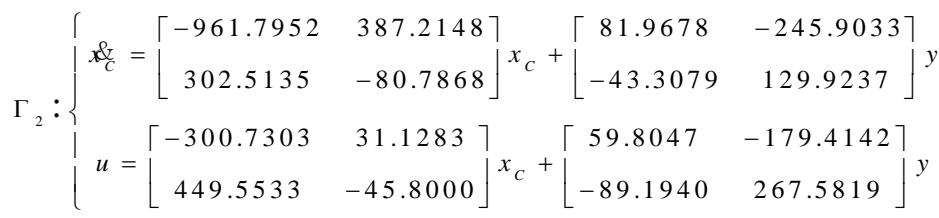

It is evident that Example 1 and example 2 are neither of the designed controllers makes the associated subsystem asymptotically stable.

\section{Conclusions}

The robust $H_{\infty}$ control problem has been studied via switched state feedback and dynamic output feedback for state delay switched linear systems with exponential uncertainties by using Taylor series approximation, convex polytope technique and LMI method. Sufficient conditions are, by solving linear matrix inequalities, presented to realize the $H_{\infty}$ control design. How to design a switched controller and associated state feedback sub-controllers to improve the performance of uncertain discrete-time switched linear systems with state delay should be further studied in the future work.

\section{Acknowledgements}

This work was supported by the Talents Foundation of Guizhou Institute of Technology (XJZK20131207), the Natural Science Foundation of China under Grant (6121630052), the Program for New Century Excellent Talents in Chinese University under Grant (NCET-12-0657).

\section{References}

[1] J. H. Ge, P. M. Frank and C. F. Lin, $H_{\infty}$ Control via Output feedback for State Delayed Systems. International Journal of Control. 64,1 (1996).

[2] E. T. Jeung, T. H. Kim, H. B. Park, $H_{\infty}$ Output feedback Controller Design for Linear Systems with Time-varying Delayed State. IEEE Trans on Automatic Control. 43,7 (1998).

[3] Z. D. Wang, B. Huang, H. Unbehauen, Robust $H_{\infty}$ Observe Design of Linear Time-delay Systems with Parametric Uncertainty. System \& Control Letters, 2001, 42, 4 (2001).

[4] Y. J.Wang, G. M. Xie and L. Wang, Controllability of Switched Time-delay Systems under Constrained Switching. Journal of Math Analysis Application. 286, 2 (2003). 
[5] G. Xie, L. Wang, Controllability of Switched Linear Discrete-time Systems with Time Delay. Proceedings of the 4th American Control Conference, (2003), Denver, USA.

[6] G. M. Xie, L. Wang, Stabilization of Switched Linear Systems with Time-delay in Detection of Switching Signal. Journal of Mathematical Analysis and Applications. 305 (2005).

[7] J. Shi, T. J. Wu, S. X. Du, Delay-dependent Robust $H_{\infty}$ Control for a class of Uncertain Switched Systems with Time Delay. Journal of Zhejiang University. 5, 7 (2004).

[8] S. Kim, S. A. Campbell, X. Z. Liu, Stability of a Class of Linear Switching Systems with Time Delay. IEEE Trans on Circuits and Systems-I; Regular papers. 53, 2 (2006).

[9] Y. G. Sun, L.Wang, G. M. Xie, Stability of Switched Systems with Time-Varying Delays:DelayDependent Common Lyapunov Functional Approach. Proceedings of the American Control Conference, (2006), Minneapolis Minnesota, USA.

[10] C. H. Wang, L. X. Zhang, H. J. Gao, et al, Delay-dependent Stability and Stabilization of a Class of Linear Switched Time-varying Delay Systems. IEEE Proc of the 4th International Conference on Machine Learning and Cybernetics, (2005), Guangzhou, China.

[11] L. Hetel, J. Daafouz, C. Iung, Stabilization of arbitrary switched linear systems with unknown time varying delays. IEEE Trans. on Automatic Control. 51, 10 (2006).

[12] L. Hetel, J. Daafouz, C. Iung, LMI control design for a class of exponential uncertain system with applications to network controlled switched systems. Proceedings of American Control Conference, (2007), July 9-13: American.

[13] B. Wittenmark, J. Nilsson and M. Törngren, Timing problems in realtime control systems. Proceedings of American Control Conference, (1995), June 21-23; American.

[14] W. Zhang, M. S. Branicky, S. M. Phillips, Stability of networked control systems. IEEE Control Systems Magazine. 21,1 (2001).

[15] B. Andrea, M. Pierpaolo, L. Alberto, S. Vincentelli, Controller synthesis on non-uniform and uncertain discrete time domains. Lecture Notes in Computer Science. 3414 (2005).

[16] Scherer, Carsten \& Weiland, Siep, Lecture Notes DISC Course on Linear Matrix Inequalities in Control. Springer-Verlag, (1999).

[17] P. Gahinet, P. Apkarian, An LMI-based Parametrization of all $H_{\infty}$ Controllers with Applications. Proceedings of the 32nd Conference on Decision and Control, (1993), San Antonio, Texas, USA.

[18] J. Wang and H. Yang, Output-feedback stabilization of Markovian jump linear systems over networks with time delays. Journal of Information and Computational Science. 8, 13 (2011).

[19] C. L. Li, F. Long and G. H. Ren, Disturbance Attenuation for a Class of Discrete-time Switched Systems with Exponential Uncertainty. Journal of the Korean Mathematical Society. 48, 4 (2011).

[20] Y. Wang, P. Shi, Q. Wang and D. Duan, Exponential H-infinity filtering for singular Markovian jump systems with mixed mode-dependent time-varying delay. IEEE Trans on Circuits and Systems-I. 60, 9 (2013).

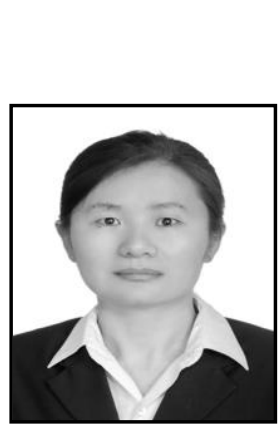

\section{Authors}

Hong-mei Huang, she is a currently lectorate of Guizhou Institute of Technology, Guiyang, China. Her research interests are in the robust control of complex system and neural network.

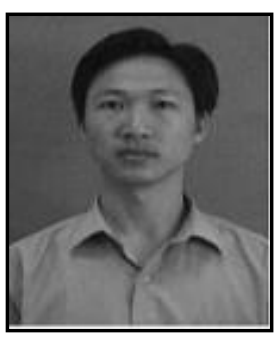

Fei Long, received the $\mathrm{Ph}$. D degree from Dongnan University, China, in 2006, and now he is a professor of Guizhou University, China. His research interests include neural network control of the complex system and intelligent information processing. 


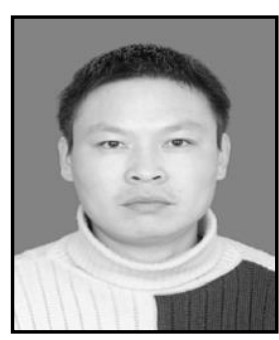

Chang-lin Li, he is an engineer of Tourism Administration of Guizhou Province, Guiyang, China. His research interests are in the robust control of linear and nonlinear systems, neural network.

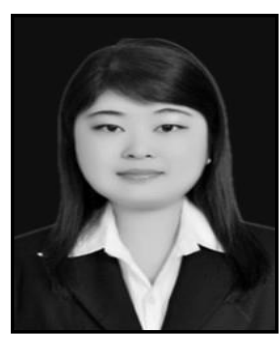

A-dang Ding, she is a teacher of Guizhou Institute of Technology, Guiyang, China. Her research interests are in the computer wireless communication and space-time coding. 